\title{
Spectral signatures of Earth's climate variability over 5 years from IASI
}

\author{
Helen Brindley, Richard Bantges, Jacqueline Russell, Jonathan Murray, Christopher Dancel,
}

\author{
Claudio Belotti and John Harries
}

Space and Atmospheric Physics Group, Imperial College, London

This is the peer reviewed version of the following article: Brindley, H, R. Bantges, J. Russell, J. Murray, C. Dancel, C. Belotti and J. Harries (2015), Spectral signatures of Earth's climate variability over 5 years from IASI, J. Climate, 28, 1649-1660, which has been published in final form at http://dx.doi.org/10.1175/JCL-D-14-00431.1.

\footnotetext{
(C) Copyright 2015 American Meteorological Society (AMS). Permission to use figures, tables, and brief excerpts from this work in scientific and educational works is hereby granted provided that the source is acknowledged. Any use of material in this work that is determined to be "fair use" under Section 107 of the U.S. Copyright Act September 2010 Page 2 or that satisfies the conditions specified in Section 108 of the U.S. Copyright Act (17 USC $\S 108$, as revised by P.L. 94-553) does not require the AMS's permission. Republication, systematic reproduction, posting in electronic form, such as on a web site or in a searchable database, or other uses of this material, except as exempted by the above statement, requires written permission or a license from the AMS. Additional details are provided in the AMS Copyright Policy, available on the AMS Web site located at (http://www.ametsoc.org/) or from the AMS at 617-227-2425 or copyrights@ ametsoc.org.
}

26 Corresponding author address: Helen Brindley, Space and Atmospheric Physics Group, Imperial

7 College London, Blackett Laboratory, Prince Consort Road, London, SW7 2AZ, UK

E-mail: h.brindley@imperial.ac.uk 


\section{Abstract}

30

31

32
Interannual variability in spectrally resolved longwave radiances is quantified at a variety of spatial scales using five years of IASI observations. Maximum variability is seen at the smallest scales investigated $\left(10^{\circ}\right.$ zonal means) at northern and southern high latitudes across the centre of the $15 \mu \mathrm{m} \mathrm{CO}$ band. As spatial scale increases, the overall magnitude of interannual variability reduces across the spectrum and the spectral shape of the variability changes. In spectral regions sensitive to conditions in the upper troposphere the effect of increasing spatial scale is relatively small and at the global scale these parts of the spectrum show the greatest yearto-year variability. Conversely the atmospheric window $(8-12 \mu \mathrm{m})$, which is sensitive to variations in surface temperature and cloud, shows a marked reduction in interannual variability with increasing spatial scale. Over the five years studied, at global scales the standard deviation in annual mean brightness temperature is less than $0.17 \mathrm{~K}$ across the spectrum; reducing to less than $0.05 \mathrm{~K}$ across the window. Spectrally integrating the IASI measurements to create pseudo broadband and window channels indicates a variation about the mean that is higher for the broadband than the window channel at the global and quasi-global scale and over the southern hemisphere. These findings are in agreement with observations from CERES Terra over the same period and imply that at the largest spatial scales, over the period considered here, fluctuations in mid-upper tropospheric temperatures and water vapour, and not cloud or surface temperature, play the dominant role in determining the level of interannual variability in all-sky outgoing longwave radiation. 
52 The potential for using measurements of spectrally resolved outgoing longwave radiation (OLR)

53 directly to monitor the climatic state and detect and attribute change has been recognised for

54 some time (e.g. Charlock et al., 1984, Kiehl et al., 1986, Goody et al., 1995, Iacono and Clough,

55 1996, Slingo and Webb, 1997, Harries et al., 2001). More recently interest in the topic has

56 revived due to the prospect of a dedicated space mission, CLARREO (Climate Absolute

57 Radiance and Refractivity Observatory), which seeks to establish high absolute accuracy

58 benchmark climate observations of spectrally resolved thermal infrared and reflected solar

59 radiation, in combination with measurements of atmospheric refraction (Wielicki et al., 2013).

60 Theoretical studies by members of the CLARREO science team have shown how distinct

61 longwave spectral signals from different climate forcing and feedback mechanisms may be

62 derived, and, importantly in the context of the temporal and spatial sampling strategy envisaged

63 for CLARREO, appear to combine with a high degree of linearity (Leroy et al., 2008, Huang et 64 al., 2010).

65 To date, attempts to evaluate changes in the forcing of climate and associated feedback processes

66 by considering changes over time to spectrally resolved OLR observations of the Earth have

67 typically been restricted to clear-sky conditions (Harries et al., 2001, Griggs and Harries, 2007).

68 These studies demonstrated that sharp spectral features due to long-term increases in

69 concentration of individual molecules such as $\mathrm{CO}_{2}, \mathrm{CH}_{4}$ and $\mathrm{CFCs}$ can be identified. However,

70 the detection of changes in the outgoing spectrum that might be due to water vapour and, in

71 particular, cloud feedback processes are much more difficult to unambiguously discern (e.g.

72 Brindley and Allan, 2003). In particular, the high level of variability in the cloud field in space

73 and time not only has implications for the interpretation of spatially and temporally averaged 
74 radiances (e.g. Kato et al., 2011), but crucially, makes disentangling responses to climate forcing

75 from underlying climate variability a hugely challenging task. Whilst there have been several

76 studies on the impact that temporal and spatial sampling may have on the accuracy with which

77 climate change signals can be detected in OLR observations (Brindley and Harries, 2003a;

78 Brindley and Harries, 2003b, Kirk-Davidoff et al., 2005), understanding the exact nature and

79 level of background variability seen in observed all-sky spectra is an important question which

80 has not yet been fully addressed.

81 Recognising this challenge, the work presented here exploits the emerging radiance record

82 available from the Infrared Atmospheric Sounding Interferometer (IASI) on the European Metop

83 satellite (Hilton et al., 2012) to investigate the interannual variability in average OLR spectra at

84 different spatial scales. Results are placed in the context of broadband observations from the

85 Clouds and the Earth's Radiant Energy System (CERES) instruments (Wielicki et al., 1996) to

86 assess both the consistency between the different records and the additional insights that can be

87 gained from the higher spectral resolution available from IASI. Hence, in section 2 we briefly

introduce IASI and CERES before focusing on the insights provided by the former instrument in

section 3. Where appropriate we also make reference to the results presented in Huang and

90 Ramaswamy (2009) who, in their efforts to assess the time evolution of spectral OLR, evaluated

91 the monthly and annual variability seen in global mean observations from the Atmospheric

92 InfraRed Sounder (AIRS) (Chahine et al., 2006) over the period 2002-2007. We show that the

93 interannual variability manifested across the IASI spectra is less than $0.17 \mathrm{~K}$ in brightness

94 temperature in the global annual mean, collapsing to a value of less than $0.05 \mathrm{~K}$ in the so-called

95 atmospheric window $\left(\sim 800-1250 \mathrm{~cm}^{-1}\right)$, a remarkable result with implications for the variability

96 of the cloud field and the land surface. In section 4 we use the observations from CERES to 
97 illustrate that these results are consistent with patterns of behaviour seen in both broadband and

98 window OLR fluxes and investigate issues related to instrument sampling. We discuss the

99 potential implications of these results, as well as the caveats associated with our study in section

1005.

101 2. Observational Tools

102 a. IASI

103 IASI is a Michelson Interferometer, covering the spectral range from 645 to $2760 \mathrm{~cm}^{-1}$ in three 104 separate wavenumber bands running from 645-1210, 1210-2000 and 2000-2760 $\mathrm{cm}^{-1}$ (Simeoni et 105 al., 2004) with an apodised half-width of $0.5 \mathrm{~cm}^{-1}$. Since October 2006 it has enabled high106 resolution atmospheric sounding from the sun-synchronous Metop-A platform which has an 107 equator crossing time of 0930 local time for the descending node. The instrument is a cross108 track scanner, producing 30 'fields of regard' (FOR) per scan; each is an array of 2 x 2 pixels 109 with a $12 \mathrm{~km}$ diameter at nadir. Instrument noise levels are found to be within specification and 110 stable over time except for wavenumbers below $680 \mathrm{~cm}^{-1}$ and in small band overlap regions 111 where observations from different detectors are merged (Blumstein et al., 2007). In the spectral 112 region of interest for this study NE $\Delta \mathrm{T}$ levels (expressed at 280K) are always below $0.4 \mathrm{~K}$ 113 (minimum wavenumber $\sim 660 \mathrm{~cm}^{-1}$ ) and more typically less than $0.3 \mathrm{~K}$. Comparisons with co114 located observations from the Atmospheric InfraRed Sounder indicate agreement between the 115 two instruments to within +/-0.2K, while calibration/validation activities using aircraft based 116 interferometers give agreement to within +/-0.3 K (Newman et al., 2008, Larar et al., 2010).

117 Here we use only 'nadir' IASI observations (in practice, within $5^{\circ}$ of nadir) covering the spectral 118 range $645-1600 \mathrm{~cm}^{-1}$ which, over the five years from January 2008 to December 2012, yields 
$119 \sim 160$ million spectra as input to the study. As neither high spatial nor spectral resolution is a 120 priority, initial data reduction to the $\sim 100 \mathrm{~km}$ spatial scale is made by averaging 16 IASI near 121 nadir spectra and the result smoothed to a $2.8 \mathrm{~cm}^{-1}$ spectral resolution, with a view to facilitating 122 comparisons with previous analyses which used data from the IRIS mission (Hanel et al., 1972) 123 in future studies. The resulting IASI reduced resolution (hereafter IRR) spectra form the basic 124 input to the averaging studies discussed in the following section and are comparable in spectral 125 and spatial detail to those used in the work of Harries et al. (2001) and Griggs and Harries 126 (2007). Figure 1 illustrates the global, annual mean IRR spectrum for 2008-2012 to place the 127 variability plots shown in later sections in context. Principal spectral features are due to $\mathrm{CO}_{2}$ $128\left(\sim 650-800 \mathrm{~cm}^{-1}\right), \mathrm{O}_{3}\left(\sim 1000-1070 \mathrm{~cm}^{-1}\right), \mathrm{CH}_{4}\left(\sim 1200-1400 \mathrm{~cm}^{-1}\right.$, centred at $\left.1303 \mathrm{~cm}^{-1}\right)$, and $\mathrm{H}_{2} \mathrm{O}$ $129\left(\sim 1250-1600 \mathrm{~cm}^{-1}\right)$, with other weaker bands also in evidence, e.g. $\mathrm{CFCl}_{3}\left(853 \mathrm{~cm}^{-1}\right)$.

130 In the large scale annual averages presented in this study at least 1.6 million native resolution 131 IASI spectra are used to obtain the final IRR results. If the source of the $0.4 \mathrm{~K} \mathrm{NE} \Delta \mathrm{T}$ error 132 indicated above was purely random in nature this would translate to a final uncertainty that is of 133 the order $3 \times 10^{-4} \mathrm{~K}$ in zonal mean averages, and a factor of $\sim 4$ smaller in the global mean. In 134 reality it is likely that there is a systematic component to the NE $\Delta \mathrm{T}$ that is not reduced by 135 averaging and cannot be estimated easily. However, since we are focussed on diagnosing 136 variability in this study, it is the stability of IASI which is paramount. Comparisons with AIRS 137 show radiometric agreement between the two instruments that is within a few $\mathrm{mK}$ or better 138 (Hilton et al., 2012 and references therein) suggesting excellent stability over time. 
Since 2001 CERES has provided global measurements of the Earth's Radiation Budget from low earth orbit (Wielicki et al., 1996). Besides contributing to numerous important studies of the

142 effects of different climate processes on the Earth's energy balance, the uncertainties associated

143 with the measurements have also been rigorously assessed and documented (e.g. Loeb et al., 144 2009). In this study we make use of observations from the Terra and Aqua platforms over the 145 same 2008-2012 period considered for IASI. The CERES instrument measures the broadband 146 shortwave radiation reflected at the top of the atmosphere (TOA) in conjunction with the total 147 outgoing longwave radiation (OLR), the latter nominally covering the range 5-100 $\mu \mathrm{m}$. In 148 addition, observations are also made in a longwave window channel sensitive to radiation 149 between approximately 8-12 $\mu \mathrm{m}$. In order to make use of these window fluxes we employ level 1503 monthly mean files provided at $1^{\circ}$ latitude-longitude resolution, derived from single-scanner 151 footprints (so called SSF1deg_Ed2.7). Note that these products are created from hourly data 152 which are obtained using temporal interpolation assuming constant meteorology. Over non snow 153 covered land, during the day a half-sine fit is used, with night-time fluxes set to a constant value 154 if the daytime flux is greater than the night-time value. Over ocean and snow, linear 155 interpolation is used between the CERES overpass times.

\section{3. Inter-annual variability in IRR spectra}

158 We begin by considering the interannual variation in IRR brightness temperature spectra at the

$15910^{\circ}$ zonal scale, as this spatial scale is consistent with the type of averages expected to be 160 produced by the CLARREO mission. Figure 2 shows the standard deviation across the 5 years 
161 in the annual-mean brightness temperatures, $\sigma_{\mathrm{TB}}$, for $10^{\circ}$ zonal bands in the northern (a) and

162 southern (b) hemisphere. Maximum $\sigma_{\mathrm{TB}} \mathrm{S}$ occur across the $15 \mu \mathrm{m} \mathrm{CO}$ band centre (from $\nu \sim$

$163645-700 \mathrm{~cm}^{-1}$ ) at northern and southern high latitudes, peaking at $\sim 690 \mathrm{~cm}^{-1}$ in these zones.

164 Noting the noise characteristics of IASI we focus our discussions on observations in excess of

$165660 \mathrm{~cm}^{-1}$. Emission to space at the very centre of the band at $667 \mathrm{~cm}^{-1}$ originates from the mid-

166 upper stratosphere. Moving away from the central peak, emission to space occurs from

167 systematically lower levels in the stratosphere, until by $\sim 680 \mathrm{~cm}^{-1}$ one is effectively sounding

168 the tropopause. As wavenumber increases over the $\mathrm{CO}_{2}$ band wing $\left(\sim 700-760 \mathrm{~cm}^{-1}\right)$, emission

169 from successively lower levels in the troposphere is observed (see Fig. 1).

170 Distinct peaks in $\sigma_{\mathrm{TB}}$ are also seen in the centre of the $1303 \mathrm{~cm}^{-1} \mathrm{CH}_{4}$ band and strong water

171 vapour lines at wavenumbers $>1500 \mathrm{~cm}^{-1}$. These are largest within the $80-90^{\circ}$ zones but are still

172 clearly apparent at lower latitudes, particularly in the southern hemisphere. Within the northern

173 hemisphere, variability within the atmospheric window region $\left(v \sim 800-1250 \mathrm{~cm}^{-1}\right)$ is typically

174 higher than that seen within the $\mathrm{CO}_{2}$ band wing (from $720-760 \mathrm{~cm}^{-1}$ ) and across the $6.3 \mu \mathrm{m}$ water

175 vapour vibration-rotation band $\left(v>1250 \mathrm{~cm}^{-1}\right)$. No consistent pattern with latitude is seen

176 within the $9.6 \mu \mathrm{m} \mathrm{O}_{3}$ band $\left(v \sim 1000-1070 \mathrm{~cm}^{-1}\right)$, although for the majority of zones the

177 variability here is higher than across the atmospheric window as a whole. We note that the

178 window region would be expected to be particularly sensitive to variations in surface

179 temperature and cloud, with the changes in spectral shape we see here potentially reflecting

180 variability in cloud microphysical properties (e.g. Strabala et al., 1994). In this study we make

181 no attempt to separate out the different factors controlling this variability but anticipate that this

182 will be an area for future investigation. 
184 Moving to larger scales, Figure 3 shows the interannual standard deviation in brightness 185 temperature for $30^{\circ}$ zones across both hemispheres. Here $30^{\circ}$ zonal mean radiances are 186 constructed from the $10^{\circ}$ zonal mean radiances employed in section 3(a), applying equal 187 weighting to each band, before converting to brightness temperature and calculating the 188 associated standard deviation. The first point to note is that, as might be expected (e.g. Brindley 189 and Harries, 2003a,b; Kirk-Davidoff et al., 2005), the overall level of $\sigma_{\mathrm{TB}}$ is decreased, falling to 190 a maximum level of $0.9 \mathrm{~K}$ (compared to $1.15 \mathrm{~K}$ ) across the $\mathrm{CO}_{2}$ band centre at southern 191 hemisphere high latitudes (Fig. 3(b)). For both hemispheres the $60-90^{\circ}$ band shows the largest $192 \sigma_{\mathrm{TB}}$ within the window region. While these values reduce rather dramatically in the southern 193 hemisphere within the lower latitude bands, the reduction in the northern hemisphere is much 194 less marked. Excepting the $9.6 \mu \mathrm{m} \mathrm{O}_{3}$ band, within the $0-30^{\circ} \mathrm{S}$ and $30-60^{\circ} \mathrm{S}$ bands variability 195 across the window is lower than other spectral regions (such as within the water vapour 196 vibration-rotation band and $15 \mu \mathrm{m} \mathrm{CO}_{2}$ band wing), behaviour that is not exhibited in any of the 197 other latitude bands.

198 Outside of the window the behaviour within the highest latitude zones is most striking.

199 Consistent with the higher spatial resolution results, maximum $\sigma_{\mathrm{TB}} \mathrm{S}$ occur across the $15 \mu \mathrm{m} \mathrm{CO}_{2}$ 200 band centre and at the peak of the $1303 \mathrm{~cm}^{-1} \mathrm{CH}_{4}$ band, particularly in the southern hemisphere. 201 The signatures of increased variability due to the strong water vapour lines at wavenumbers 202 greater than $1500 \mathrm{~cm}^{-1}$ are also still apparent within these zones. 
204 Focusing now on the largest spatial scale, Figure 4(a) shows the differences from the mean 205 spectrum shown in Fig. 1 for each year from 2008-2012. Immediately apparent is the degree of 206 stability over the five years sampled here, with year-to-year differences of less than $0.3 \mathrm{~K}$ across 207 the spectral region covered (equivalent to radiance differences of $<0.3 \mathrm{~mW} \mathrm{~m}^{-2} \mathrm{sr}^{-1}$ ). This level 208 of stability translates to the standard deviations in spectrally resolved global annual mean 209 brightness temperature shown in Figure 4(b). Similar to section 3(b), annual global mean 210 radiance spectra were constructed from the $10^{\circ}$ zonal means of section 3(a), applying the 211 appropriate area (cosine) weighting to each band, prior to conversion to brightness temperature 212 and calculation of standard deviation.

213 Across the entire spectral region the interannual global $\sigma_{\mathrm{TB}} \mathrm{s}$ are very small, at less than $0.17 \mathrm{~K}$. 214 While the reduction in overall variability with increasing spatial scale might be anticipated, the 215 impact of global averaging alters with spectral region, resulting in a distinct change in spectral 216 shape. Figure 4(b) indicates that at the global scale, rather than peaking in the $15 \mu \mathrm{m}_{2}$ band 217 centre, variability peaks within the $\mathrm{CO}_{2}$ band wing $\left(700-740 \mathrm{~cm}^{-1}\right)$ and at the centre of the 1303 $218 \mathrm{~cm}^{-1} \mathrm{CH}_{4}$ band. Note that because of the non-linearity of the transformation from radiance to 219 brightness temperature the impact of the variation on outgoing energy is larger in the former 220 spectral region. Enhanced variability is also seen beyond $1350 \mathrm{~cm}^{-1}$ within the $6.3 \mu \mathrm{m} \mathrm{H}_{2} \mathrm{O}$ 221 vibration-rotation band with a further, secondary peak in $\sigma_{\mathrm{TB}}$ apparent across the $9.6 \mu \mathrm{m} \mathrm{O}_{3}$ 222 band. Surprisingly, perhaps, in the context of Figures 2 and 3, the lowest level of variability is 223 seen within the atmospheric window (excluding the $\mathrm{O}_{3}$ band) at less than $0.05 \mathrm{~K}$. 
224 Returning to Fig 4(a) it is interesting to note that removing one year (2010) from the analysis can

225 markedly affect the magnitude of the interannual variability in some spectral regions and hence

226 the spectral shape. The early months of 2010 were associated with a strong El Niño (large

227 positive multivariate El Niño Southern Oscillation index (MEI) values) before transitioning to an

228 even stronger La Niña in the summer. During El Niño conditions one typically sees positive

229 anomalies in OLR even at the global scale, with the converse being true for a La Niña phase (e.g.

230 Loeb et al., 2012, Susskind et al., 2012). The positive brightness temperature differences seen

231 during 2010 across the majority of the spectrum would suggest that enhanced planetary emission

232 has in some sense 'won' over the course of the year. What is particularly noteworthy is the peak

233 seen across the $1303 \mathrm{~cm}^{-1} \mathrm{CH}_{4}$ band and the somewhat enhanced positive difference in the $\mathrm{CO}_{2}$

234 band wing. Both are indicative of anomalously warm mid-upper tropospheric temperatures.

235 With 2010 removed, anomalies from the 4 year $(2008,2009,2011,2012)$ mean are significantly

236 reduced $(<0.15 \mathrm{~K})$, with a peak associated standard deviation of less than $0.1 \mathrm{~K}$ (Fig 4(b)). In

237 this case, although the window region still shows minimum variation, the peak brightness

238 temperature variability is no longer seen at $1303 \mathrm{~cm}^{-1}$, but rather across the $6.3 \mu \mathrm{m} \mathrm{H}_{2} \mathrm{O}$

239 vibration-rotation band at wavenumbers $>1400 \mathrm{~cm}^{-1}$, within the $9.6 \mu \mathrm{m} \mathrm{O}_{3}$ band, and both

240 within the $15 \mu \mathrm{m} \mathrm{CO}$ band wing and at the very centre of the band. While the full 2008-2012

241 period considered here does sample a relatively wide range of MEI values, these results indicate

242 how the magnitude and spectral shape of estimates of variability are themselves critically

243 dependent on the period sampled, particularly with respect to major global and regional events

244 such as El Niño.

245 With this in mind it is informative to compare Figure 4(b) to the results shown in Figure 3(a) of

246 Huang and Ramaswamy (2009) who carried out a similar analysis using AIRS observations from 
247 2002-2007. In their work the magnitude of global annual mean variability across the same

248 spectral region as considered here does not exceed $0.16 \mathrm{~K}$, but peaks in the centre of the $15 \mu \mathrm{m}$

$249 \mathrm{CO}_{2}$ band. While a secondary peak is seen in the $\mathrm{CO}_{2}$ band wing, the relative variability seen

250 across the window and the $1250-1500 \mathrm{~cm}^{-1}$ wavenumber range is of a different sense to that seen

251 here, with larger variability in the former region compared to the latter. In addition, the

252 variability across the window manifested in the AIRS measurements is also slightly larger than

253 that seen here, reaching $\sim 0.07 \mathrm{~K}$. Results for smaller spatial scales are not presented by Huang

254 and Ramaswamy but it is interesting to note that for the majority of the period they considered

255 the climate system was in an El Niño phase. Moreover, the range in MEI values was smaller

256 than that seen over the 2008-2012 period.

\section{4. Comparison to broadband observations}

a. Window, non-window and broadband variability as a function of spatial scale

259 In this section we seek to investigate whether the spectrally resolved results seen in section 3 are

260 consistent with spectrally integrated observations made over the same 5 year period. To this end

261 we employ CERES Terra and Aqua broadband OLR and window fluxes. CERES window fluxes

262 cover the range $\sim 833-1250 \mathrm{~cm}^{-1}$ so by comparing them to the corresponding OLR measurements

263 we can assess whether a reduction in window relative to non-window interannual variability is

264 seen as spatial scale increases, as might be anticipated from the IRR results. In addition, by

265 spectrally integrating the IRR data over the appropriate wavenumber range we can perform a

266 direct comparison with IRR window radiances. For completeness, a similar integration has also

267 been performed over the full $660-1600 \mathrm{~cm}^{-1}$ range studied here to create 'broadband' IRR

268 radiances. However, these do not sample the energetically important far infra-red region (Sinha 
and Harries, 1995; Harries et al., 2008), the majority of which is captured by the CERES OLR

270 fluxes. Because of the different metrics being compared, the variability is presented in terms of

271 coefficient of variation (CV) which is simply the inter-annual standard deviation, $\sigma$, divided by

272 the five year annual mean for each dataset, $\mu$, expressed as a percentage (Eq. (1)).

$273 \quad C V=100 \times \frac{\sigma}{\mu}$

274 In addition, it should be noted that:

$275 \quad \mu_{B B}=\mu_{w i n}+\mu_{n w}$ and $\sigma_{B B}=\sqrt{\sigma_{w i n}^{2}+\sigma_{n w}^{2}+2 \operatorname{cov}(\text { win }, n w)}$

276 where BB, win and nw subscripts refer to the broadband, window and non-window region

277 respectively, and $\operatorname{cov}($ win,nw) is the covariance between the window and non-window regions.

278 Figure 5(a) shows the interannual variability in CERES Terra and Aqua window channel fluxes

279 as a function of latitude. Superimposed is the equivalent CV in IRR window radiances. It is

280 clear that all three instruments show a very similar pattern of behaviour, with minimum window

281 variability in the southern hemisphere low and mid-latitudes. It is also clear that the variability

282 about the mean for all three datasets is very low, typically less than $1 \%$. Peak variability is seen

283 in the $70-90^{\circ}$ latitude bands with, in the southern hemisphere, larger variability being manifested

284 in the CERES measurements. In the northern high latitudes the pattern switches such that IRR

285 variability is typically higher. For a given latitude band, the difference between the CVs

286 calculated for each instrument is generally less than $0.05 \%$. However, three bands display

287 noticeably higher discrepancies between the instruments: $70-80^{\circ} \mathrm{S}$ and $0-10^{\circ} \mathrm{N}$ where the IRR CV

288 is lower than that of the two CERES instruments, and $80-90^{\circ} \mathrm{N}$ where the opposite is true. 
Detailed analysis of these bands has not identified any obviously anomalous behaviour for the two CERES instruments or for IASI and it should be noted that the latitudinal pattern is still consistent between the three instruments. Figure 5(a) thus gives confidence that, despite the differing overpass times and instrument characteristics of CERES Terra, Aqua and IASI, the general pattern and level of interannual variability exhibited in window fluxes and radiances sampled by the three instruments over the five years considered here is similar. Figure 5(b) shows how the relative level of variability in the different spectral ranges changes with scale. In section 3 the analysis of IRR spectra showed that as one moved from smaller to larger spatial scales the variation in the window region typically reduced more rapidly than in spectral regions outside of the window. Figure 5(b) confirms that this behaviour is also captured in the spectrally integrated IRR data and for the two CERES instruments. Whilst variability always reduces as the scale increases, at the 10 and $30^{\circ}$ scale for all three instruments the mean window $\mathrm{CV}$ exceeds the equivalent non-window and broadband CVs by some margin. However, globally the non-window $\mathrm{CV}$ is similar in value or exceeds both the window and broadband CVs. This greater reduction in the window variability with spatial scale indicates that non-window variability becomes increasingly important and is most significant for the global average.

Furthermore, the influence of variability in spectral regions not sampled by the IRR broadband radiances $\left(v<660 \mathrm{~cm}^{-1}, v>1600 \mathrm{~cm}^{-1}\right)$ is more significant at the global scale. Recall that the $\mathrm{CV}$ is the interannual standard deviation divided by the five year annual mean. At the 10 and $30^{\circ}$ scales the larger spectral range of the CERES compared to the IRR broadband must lead to a greater increase in the mean for CERES when moving from window to broadband channel. The results show that this increase is not compensated sufficiently by the additional variability found at wavenumbers below 660 and above $1600 \mathrm{~cm}^{-1}$. Thus the broadband CERES CVs are 
312 systematically smaller than the IRR cases. However for the global average the broadband CVs

313 are similar for all three instruments indicating that at this scale the variability in the spectral

314 regions not sampled by the IRR broadband is sufficient to compensate for their additive effect on

315 the mean. This hints at an important role for the far infra-red region of the spectrum in

316 determining all-sky OLR variability at the global scale.

\section{7 b. Role of land/ocean sampling}

318 In this section we provide an indication of the potential impact of spacecraft sampling patterns

319 on the relative level of inter-annual variability seen between the window and broadband OLR.

320 CERES Terra and Aqua are in sun-synchronous orbits with nominal ascending/descending local

321 equator crossing times of $10.30 / 22.30$ and $13.30 / 01.30$ respectively. IASI is also in a sun-

322 synchronous orbit, with a nominal local equator crossing time of 09.30 (21.30). While the

323 annual means created from IASI are representative purely of the conditions at the observation

324 time, as noted in section 2.2, the CERES means will contain the effects of temporal interpolation

325 assuming constant meteorology between the instrument overpasses. We note however that using

326 the Geostationary Enhanced 'SYN1deg' CERES products does not markedly affect the results

327 reported here, suggesting that a more explicit representation of the diurnal cycle, using the

328 observations made by the individual CERES instruments as anchor points, does not influence

329 OLR variability at the spatial and temporal scales considered here.

330 The ability of a sun-synchronous orbit to truly capture the annual mean brightness temperature

331 and its variability from year to year will depend on its overpass time due to a susceptibility to the

332 phase of the diurnal cycle. Effects of this type are discussed in detail by Kirk-Davidoff et al.

333 (2005) in the context of smaller, 15 by $30^{\circ}$ grid box averages. They use 3 hourly $11 \mu \mathrm{m}$ 
334 brightness temperatures, BT11, from the Global Cloud Imagery project (Salby et al., 1991) to

assess the effects of different sampling strategies on the associated sampling error. Most pertinently in the context of this study, they show that, although a sun-synchronous orbit will never represent the optimal solution for capturing the true diurnal behaviour, a $10 \mathrm{am} / \mathrm{pm}$ equator crossing time would be expected to minimise the error seen from this type of orbit and that the year to year bias errors are highly correlated. Nonetheless, they note that interannual variability in the diurnal cycle will introduce an additional component of variability into estimates of the mean made from sun-synchronous data. We might expect that these effects would be largest in locations where the magnitude of the diurnal cycle is largest. In addition, they should be manifested in spectral regions that are most sensitive to these variations.

Figure 6(a) shows the relative magnitude of window and broadband CV for each instrument for a variety of spatial regions of intermediate scale. In the northern hemisphere, tropics and deep tropics, the ratio of window to broadband CV is very similar for the two CERES instruments and systematically smaller for IASI. This difference is consistent with the results presented in Figure 5(b) and the associated discussion. However, over the southern hemisphere there is a marked discrepancy between Terra and Aqua, with the former showing a much reduced ratio relative to the latter. The equivalent IASI ratio is intermediate between the two values. Investigation of the contributing factors indicates that it is the window channel standard deviation which is responsible for this behaviour, being approximately halved for Terra relative to Aqua when considering the southern hemisphere as a whole. Given the land-ocean distribution we might expect any sensitivity to interannual variability in the diurnal cycle (and hence overpass time) to be more marked in the northern hemisphere so this result is rather surprising. What is more consistent with expectation is the reduction in window standard deviation seen between the 
357 northern and southern hemisphere for all instruments, which is reflected in the lower CV ratios

358 over the southern hemisphere.

359 This reduction in window to broadband CV ratio over the southern hemisphere, coupled with the 360 very low window standard deviations seen in oceanic bands (e.g. Fig. 2(b): 50-60 S) does

361 indicate that instrument sampling will influence the exact magnitude and spectral distribution of 362 variability, even at the extended temporal and spatial scales considered here. Figure 6(b) is an 363 attempt to illustrate this more clearly using the IRR observations. Here the data have been sub364 divided into land, ocean, day and night sub-categories prior to averaging. For all regions the 365 window relative to the broadband CV is enhanced when only land scenes are considered. The 366 most spectacular ocean/land differences are seen for the northern hemisphere case, where a 367 negative covariance between the window and non window parts of the spectrum results in very 368 low broadband standard deviations over land. More typically, the window and broadband 369 standard deviations over land are both enhanced relative to those seen over ocean but the 370 fractional increase relative to the mean is larger for the window channel. Restricting each region 371 to ocean scenes only, the window $\mathrm{CV}$ is always comparable to, or smaller than, the equivalent 372 broadband value.

\section{5. Discussion and Conclusions}

374 In this study we have used five years of IASI observations to assess the level of inter-annual 375 variability seen within the Earth's outgoing longwave radiation spectrum (from $660-1600 \mathrm{~cm}^{-1}$ ) 376 at a variety of spatial scales ranging from $10^{\circ}$ zonal means to global averages. Our results

377 indicate that on these timescales, peak interannual variability at the smallest spatial scales is seen 378 across the centre of the $15 \mu \mathrm{m} \mathrm{CO}_{2}$ band $\left(660-690 \mathrm{~cm}^{-1}\right)$ at high latitudes. At the very centre of 
379 the band $\left(\sim 667 \mathrm{~cm}^{-1}\right)$ this is likely a reflection of variation in planetary wave activity and, 380 particularly in the northern hemisphere, the effects of sudden stratospheric warmings (e.g.

381 Charlton and Polvani, 2007). The variability at wavenumbers between $\sim 670-690 \mathrm{~cm}^{-1}$, sounding 382 the tropopause, is consistent with the fact that the seasonal cycle in tropopause temperature peaks 383 over southern hemisphere high latitudes with a secondary maximum over the Arctic, and can 384 show significant inter-annual variability over both locations (e.g. Kishore et al., 2006).

385 As spatial scale increases the interannual variability reduces across the spectrum, but this 386 reduction occurs at a different rate for different spectral regions. While the interannual 387 variability within the atmospheric window, most sensitive to surface temperature and cloud, 388 reduces relatively rapidly with scale, variability in areas of the spectrum sensitive to mid-upper 389 troposphere temperature and water vapour shows a slower reduction. As a consequence, at the 390 global scale, interannual variability peaks in the wing of the $15 \mu \mathrm{m} \mathrm{CO}_{2}$ band, across the 1303 $391 \mathrm{~cm}^{-1} \mathrm{CH}_{4}$ band, and within the $6.3 \mu \mathrm{m}$ water vapour vibration-rotation band at wavenumbers 392 greater than $1400 \mathrm{~cm}^{-1}$. At this global scale, interannual variability across the entire spectrum is 393 less than $0.17 \mathrm{~K}$, reducing to less than $0.05 \mathrm{~K}$ across the window. These values translate to 394 equivalent radiance values of 0.17 and $0.05 \mathrm{~mW} \mathrm{~m}^{-2} \mathrm{~cm} \mathrm{sr}^{-1}$ and highlight the remarkable 395 stability of longwave emission from our planet as a whole. Similar findings in terms of the 396 magnitude of spectral variability were reported by Huang and Ramaswamy (2009) based on 397 analysis of AIRS data from 2002-2007. However, in that work the spectral shape of the 398 variability was different to what we obtain here from IASI. It is an open question as to whether 399 this change in shape is a result of the different periods covered, the different overpass times of 400 the satellites carrying AIRS and IASI, or a result of instrument performance. Nonetheless, 401 results of this type put observationally based limits on how the Earth's spectral emission to space 
402 varies interannually on the global scale, an important constraint which can be used to test and 403 improve climate models.

404 Using broadband and window fluxes from the CERES instruments on Terra and Aqua we have 405 shown that as spatial scale increases the OLR displays a similar reduction in inter-annual 406 variability and is consistent with the spectral analysis in how this scaling behaviour differs in 407 different spectral regions. At the smallest scales, the percentage variation about the mean (the 408 coefficient of variation, CV) is substantially larger for the window channel compared to the 409 broadband. As scale increases the $\mathrm{CV}$ in each spectral regime becomes more equivalent until at 410 the global scale, for CERES Terra at least, the broadband CV exceeds that seen in the window 411 channel. This behaviour arises because of the increasing importance of the variation from 412 spectral regions outside of the window to the total broadband variance. Equivalent behaviour is 413 seen in suitably spectrally integrated IASI observations.

414 Previous work has illustrated a strong anti-correlation between cloud and surface temperature 415 variations within the tropics which may result in a compensation effect across the atmospheric 416 window at the global scale (e.g. Huang and Ramaswamy, 2008). While OLR across the 417 remainder of the longwave spectrum would also be affected by the presence of cloud, the impact 418 of low and mid-level cloud across much of the spectrum is strongly damped by overlying upper 419 tropospheric water vapour. It is feasible that high level cloud and surface temperature variations 420 compensate each other in such a way that there is a minimal signal across the window coupled 421 with enhanced variability in the $\mathrm{CO}_{2}$ band wing and water-vapour vibration-rotation band.

422 However, we have performed a set of realistic spectral simulations (to be reported in a follow on 423 study) that do not show this behaviour. It also appears inconsistent with the findings of Huang 424 and Ramaswamy (2009). There, much larger changes are seen across the window than within 
425 the water vapour bands or $\mathrm{CO}_{2}$ band wing when comparing all-sky to clear-sky conditions.

426 Hence, at the global and quasi-global scale we argue that our results imply that it is fluctuations

427 in mid-upper tropospheric temperatures and water vapour, and not cloud or surface temperature,

428 that play the dominant role in determining the level of inter-annual all-sky OLR variability, at

429 least over the period we have considered here.

430 Given current interest in developing climate monitoring missions that will explicitly use

431 spectrally resolved information it is interesting to note the potential effects of sampling and

432 record length on the robustness of our findings. A reliable estimate of background variability is

433 a key tool for determining whether a particular spectral signal is indicative of a true change or

434 simply a climate fluctuation, and to identify which spectral regions offer the most promise for

435 rapid change detection (e.g. Goody et al., 1995). Hence, factors that can perturb both the

436 magnitude and spectral shape of the background variability need to be understood and their

437 effects quantified.

438 Decomposing the IASI results further has allowed us to provisionally probe the effects of

439 sampling on our results. Splitting the data into land and ocean, and day and night categories

440 indicates that enhanced variability is seen over land scenes, manifested through the magnitude

441 of, in particular, window channel interannual standard deviations. This effect means that, even at

442 the global scale, IASI window radiances show a larger CV than their broadband counterparts

443 when only land scenes are averaged. Conversely, when only ocean scenes are included in the

444 spatial averaging the window CV falls below that seen for the broadband for a number of regions

445 including the deep-tropics, tropics and both hemispheres. These findings suggest that the

446 precise satellite overpass time will affect both the magnitude and shape of any temporally and

447 spatially averaged spectra that are produced. Similar conclusions can be drawn regarding the 
448 effects of record length on the variability exhibited within that record. While these results

449 indicate the care that must be attached to interpreting the results from a given satellite record,

450 previous work suggests that for instruments in sun-synchronous orbit, an early morning equator

451 crossing time, similar to that of both IASI and CERES Terra will minimise diurnal sampling

452 errors (Kirk-Davidoff et al., 2005). When all scenes are included in this study a smaller CV for

453 the window compared to broadband is seen for IASI for the southern hemisphere, quasi-global

454 and global mean cases, and this pattern of behaviour is replicated by CERES Terra. Similarly

455 even when individual years are excluded from the period of study, minimum variability at the

456 global scale is still seen across the atmospheric window.

457 It should be noted that in the analysis presented here there is no attempt to account for any

458 trends, either real, or arising as a result of instrument performance, in the IRR data. There have

459 certainly been real, monotonic increases in the annual mean concentrations of certain greenhouse

460 gases (e.g. $\mathrm{CO}_{2}, \mathrm{CH}_{4}$ ) over the 2008-2012 time period and these would be expected to enhance

461 absorption in those regions of the OLR spectrum sensitive to their presence. Acting in isolation

462 this increased absorption would drive the OLR in a consistent direction with time, however, in

463 reality any response will be modified by the temperature variability over the vertical levels where

464 the absorption is taking place. Figure 4(a) suggests that no consistent linear trend in spectral

465 OLR as measured by IASI exists with time across the wavenumber range considered here, at

466 least at the global, annual mean scale. Turning to instrumental effects, while the absolute

467 accuracy of the measurements cannot be quantified, studies have shown that the radiometric

468 performances of IASI and AIRS agree to within a few $\mathrm{mK}$ or less as a function of time (Hilton et $469 a l ., 2012)$ implying excellent temporal stability. 
470 There is clearly a substantial amount of further information that can be extracted from the IASI

471 data. In the short-term work is ongoing to establish whether the variability highlighted here is

472 replicated in atmospheric reanalysis datasets and, if so, what processes drive this behaviour.

473 Plans are in place to perform a detailed comparison with measurements from the AIRS

474 instrument spanning the same time period, and the IASI data will also allow a re-evaluation of

475 previous analyses comparing spectra measured in 1970 by the IRIS instrument on Nimbus-4

476 (Hanel et al., 1972) to assess whether it is possible to unambiguously identify 40 year plus

477 change signals in all-sky data from the two instruments.

478 Acknowledgements

479 CERES data were extracted from http://ceres.larc.nasa.gov/order_data.php. IASI L1c data were 480 obtained from the NERC Earth Observation Data Centre (NEODC). RJB, CD and CB were 481 supported by the National Centre for Earth Observation (NCEO).

\section{References}

483 Blumstein, D., B. Tournier, F. Cayla, T. Phulpin, R. Fjortoft, C. Buil and G. Ponce, 2007, In484 flight performance of the infrared atmospheric sounding interferometer (IASI) on METOP-A. 485 Proceedings of SPIE, 6684, H6840-H6840

486 Brindley, H. and R. Allan, 2003, Simulations of the effects of interannual and decadal variability 487 on the clear-sky outgoing longwave radiation spectrum. Q. J. R. Meteorol. Soc., 129, 2971-2988

488 Brindley, H. and J. Harries, 2003a, The impact of instrument field of view on measurements of 489 cloudy-sky spectral radiances from space: application to IRIS and IMG, J. Quant. Spect. Rad. 490 Trans., 78, 341-352 
491 Brindley, H. and J. Harries, 2003b, Observations of the infrared outgoing spectrum of the Earth

492 from space: The effects of temporal and spatial sampling, J. Clim., 16, 3820-3833

493 Chahine, M., and Coauthors, 2006, AIRS: Improving weather forecasting and providing new 494 data on greenhouse gases. Bull. Amer. Meteor. Soc., 87, 911-926

495 Charlock, T., 1984, $\mathrm{CO}_{2}$ induced climatic-change and spectral variations in the outgoing 496 terrestrial infrared radiation. Tellus- $B, \mathbf{3 6}, 139-148$

497 Charlton, A. and L. Polvani, 2007, A new look at stratospheric sudden warmings. Part 1: 498 Climatology and modeling benchmarks. J. Clim., 20, 449-469

499 Goody, R., R. Haskins, W. Abdou and L. Chen, 1995, Detection of climate forcing using 500 emission spectra. Earth Observation and Remote Sensing, 13, 713-722

501 Griggs, J. and J. Harries, 2007, Comparison of spectrally resolved outgoing longwave radiation 502 over the tropical Pacific between 1970 and 2003 using IRIS, IMG and AIRS. J. Climate, 20, $503 \quad 3982-4001$

504 Hanel, R., B. Conrath, V. Kunde, C. Prabhakara, I. Revah, V. Salomonson and G. Wolford, 505 1972, The Nimbus 4 infrared spectroscopy experiment,1. Calibrated thermal emission spectra, $J$ 506 Geophys Res., 77, 2629-2641

507 Harries, J., H. Brindley, P. Sagoo and R. Bantges, 2001, Increases in greenhouse forcing inferred 508 from the outgoing longwave spectra of the Earth in 1970 and 1997. Nature, 410, 355-357

509 Harries, J., B. Carli, R. Rizzi, C. Serio, M. Mlynczak, L. Palchetti, T. Maestri, H. Brindley, and 510 G. Masiello, 2008, The Far-infrared Earth, Rev. Geophys., 46, doi:10.1029/2007RG000233 
511 Hilton, F. and Coauthors, 2012, Hyperspectral Earth observation from IASI: five years of 512 accomplishments. Bull. Amer. Meteor. Soc., doi: 10.1175/BAMS-D-11-00027.1

513 Huang, Y. and V. Ramaswamy, 2008, Observed and simulated seasonal co-variations of 514 outgoing longwave radiation spectrum and surface temperature. Geophys. Res. Lett., 35, 515 doi:10.1029/2008GL034859

516 Huang, Y. and V. Ramaswamy, 2009, Evolution and Trend of the Outgoing Longwave Radiation 517 Spectrum. J. Climate, 22, 4637-4651

518 Huang, Y., S. Leroy, P. J. Gero, J. Dykema, and J. Anderson, 2010, Separation of longwave 519 climate feedbacks from spectral observations. J. Geophys. Res, 115, doi:10.1029/2009JD012766 520 Iacono, M. and S. Clough, 1996, Application of infrared spectrometer clear sky spectral radiance 521 to investigations of climate variability. J. Geophys., Res., 101, 29439-29460

522 Kato, S., B. Wielicki, F. Rose, X. Liu, P. Taylor, D. Kratz, M. Mlynczak, D. Young, N. 523 Phojanamongkolkii, S. Sun-Mack, W. Miller and Y. Chen, 2011, Detection of atmospheric 524 changes in spatially and temporally averaged infrared spectra observed from space. J. Clim., 24, $525 \quad 6392-6407$

526 Kiehl, J., 1986, Changes in the radiative balance of the atmosphere due to increases in $\mathrm{CO}_{2}$ and 527 trace gases. Adv. Space Res., 6, 55-60

528 Kirk-Davidoff, D., R. Goody and J. Anderson, 2005, Analysis of sampling errors for climate 529 monitoring satellites. J. Clim., 18, 810-822 
530 Kishore, P., S. Namboothiri, K. Igarashi, J. Jiang, C. Ao and L. Romans, 2006, Climatological 531 characteristics of the tropopause parameters derived from GPS/CHAMP and GPS/SAC-C 532 measurements. J. Geophys. Res., 111, doi:10.1029/2005JD006827

533 Larar, A., W. Smith, D. Zhou, H. Revercomb, J. Taylor, S. Newman and P. Schlussel, 2010, 534 IASI spectral radiance validation inter-comparisons: case study assessment from the JAIVEx 535 field campaign. Atmos. Chem. Phys., 10, 411-430

536 Leroy, S., J. Anderson, J. Dykema, and R. Goody, 2008, Testing climate models using thermal 537 infrared spectra. J. Climate, 21, 1863-1875

538 Loeb, N., B. Wielicki, D. Doelling, G-L. Smith, D. Keyes, S. Kato, N. Manolo-Smith, T. Wong, 539 2009, Toward optimal closure of the Earth's Top-of-Atmosphere radiation budget, J. Climate, 22, $540 \quad 748-766$

541 Loeb, N., J. Lyman, G. Johnson, R. Allan, D. Doelling, T. Wong, B. Soden, and G. Stephens, 542 2012, Observed changes in top-of-the-atmosphere radiation and upper-ocean heating consistent 543 within uncertainty. Nature Geoscience, 15, 110-113

544 Newman, S., F. Hilton, J. Taylor, A. Collard, W. Smith and A. Larar, 2008, Direct radiance 545 validation of IASI-Results from JAIVEx. Tech. Proc. 16th Int. TOVS Study Conf., Angra Dos 546 Reis, Brazil, International TOVS Working Group. [Available online at 547 http://cimss.ssec.wisc.edu/itwg/itsc/itsc16/presentations/-2_08_newman.pdf]

548 Salby, M., H. Hendon, K. Woodberry and K. Tanaka, Analysis of global cloud imagery from 549 multiple satellites. Bull. Amer. Meteor. Soc., , 467-480 
550 Simeoni, D., and 15 co-authors, 2004, Design and development of IASI instrument, Proceedings

551 of SPIE, 5543, 208-219

552 Sinha, A. and J. Harries, 1995, Water-vapor and greenhouse trapping - the role of far-infrared

553 absorption, Geophys. Res Lett., 22, 2147-2150

554 Slingo, A. and M. Webb, 1997, The spectral signature of global warming. Q. J. R. Meteorol.

555 Soc., 123, 293-307

556 Strabala, K., S. Ackerman and W. Menzel, 1994, Cloud properties inferred from 8-12-mu-m

557 data. J. Appl. Meteorol., 33, 212-229.

558 Susskind, J., G. Molnar, L. Iredell and N. Loeb, 2012, Interannual variability of outgoing

559 longwave radiation as observed by AIRS and CERES. J. Geophys. Res., 117, doi:

$560 \quad 10.1029 / 2012 J D 017997$

561 Wielicki, B. and 39 co-authors, 2013, Achieving climate change accuracy in orbit, Bull. Amer.

562 Meteor. Soc., 94, 1519-1539 
565 Figure 1: Average 2008-2012 global, annual mean IRR brightness temperature spectrum.

566 Vertical dashed lines at $700 \mathrm{~cm}^{-1}$ and $1303 \mathrm{~cm}^{-1}$ are included to help orientate the reader in the

567 discussions concerning spectral features and variability contained in the main text

568

569 Figure 2: Standard deviation in $10^{\circ}$ zonal, annual mean all-sky IRR brightness temperature

570 spectra for the northern (a) and southern hemisphere (b). Vertical dashed lines are provided at

571700 and $1303 \mathrm{~cm}^{-1}$

572

573 Figure 3: As Figure 1 for standard deviation in $30^{\circ}$ zonal annual mean spectra

574

575 Figure 4: (a) Deviation in annual global mean brightness temperature from the five year average 576 global annual mean spectrum shown in Fig 1; (b) Standard deviation in global annual mean IRR

577 brightness temperatures for all 5 years (black) and excluding 2010 (red). Dashed vertical lines as 578 in Figures 1 to 3

579

580 Figure 5: (a) Coefficient of Variation (CV) in $10^{\circ}$ annual zonal mean window fluxes and 581 radiances measured by CERES and IASI respectively as a function of latitude. (b) Dependence 582 of $\mathrm{CV}$ on spatial scale for different spectral regions and instruments

583

584 Figure 6: Ratio of window to broadband CV for selected regions for (a) Terra, Aqua and IASI 585 (IRR spectra); (b) IASI as a function of sampling characteristics. (Deep-Tropics: $10^{\circ} \mathrm{S}-10^{\circ} \mathrm{N}$;

586 Tropics: $30^{\circ} \mathrm{S}-30^{\circ} \mathrm{N}$; NH: 0-90 $\mathrm{N}$; SH 0-90 $\mathrm{S}$; Quasi Global: $60^{\circ} \mathrm{S}-60^{\circ} \mathrm{N}$ ) 


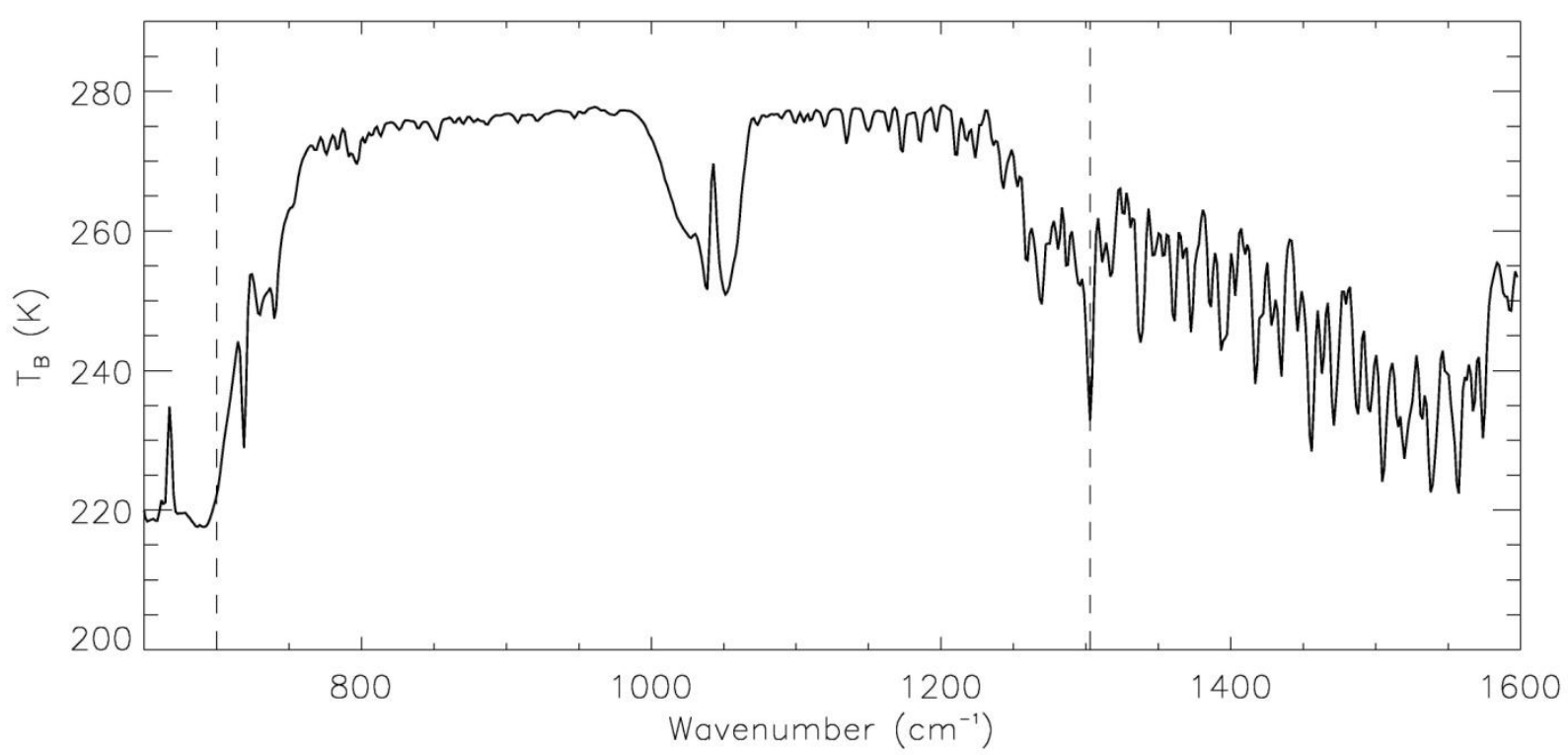

587

588 Figure 1: Average 2008-2012 global, annual mean IRR brightness temperature spectrum.

589 Vertical dashed lines at $700 \mathrm{~cm}^{-1}$ and $1303 \mathrm{~cm}^{-1}$ are included to help orientate the reader in the 590 discussions concerning spectral features and variability contained in the main text. 

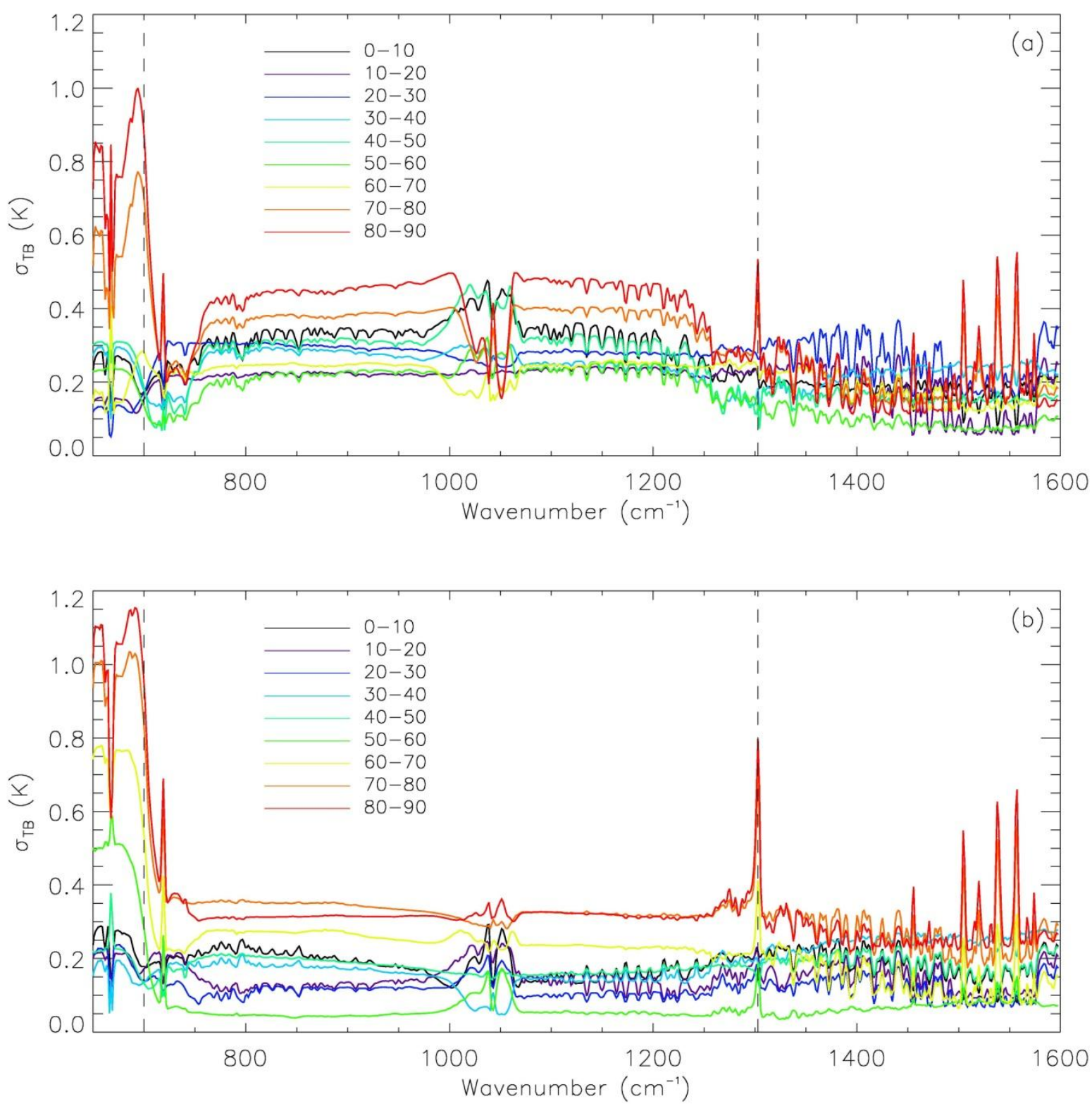

594 Figure 2: Standard deviation in $10^{\circ}$ zonal, annual mean all-sky IRR brightness temperature

595 spectra for the northern (a) and southern hemisphere (b). Vertical dashed lines are provided at 596700 and $1303 \mathrm{~cm}^{-1}$ 

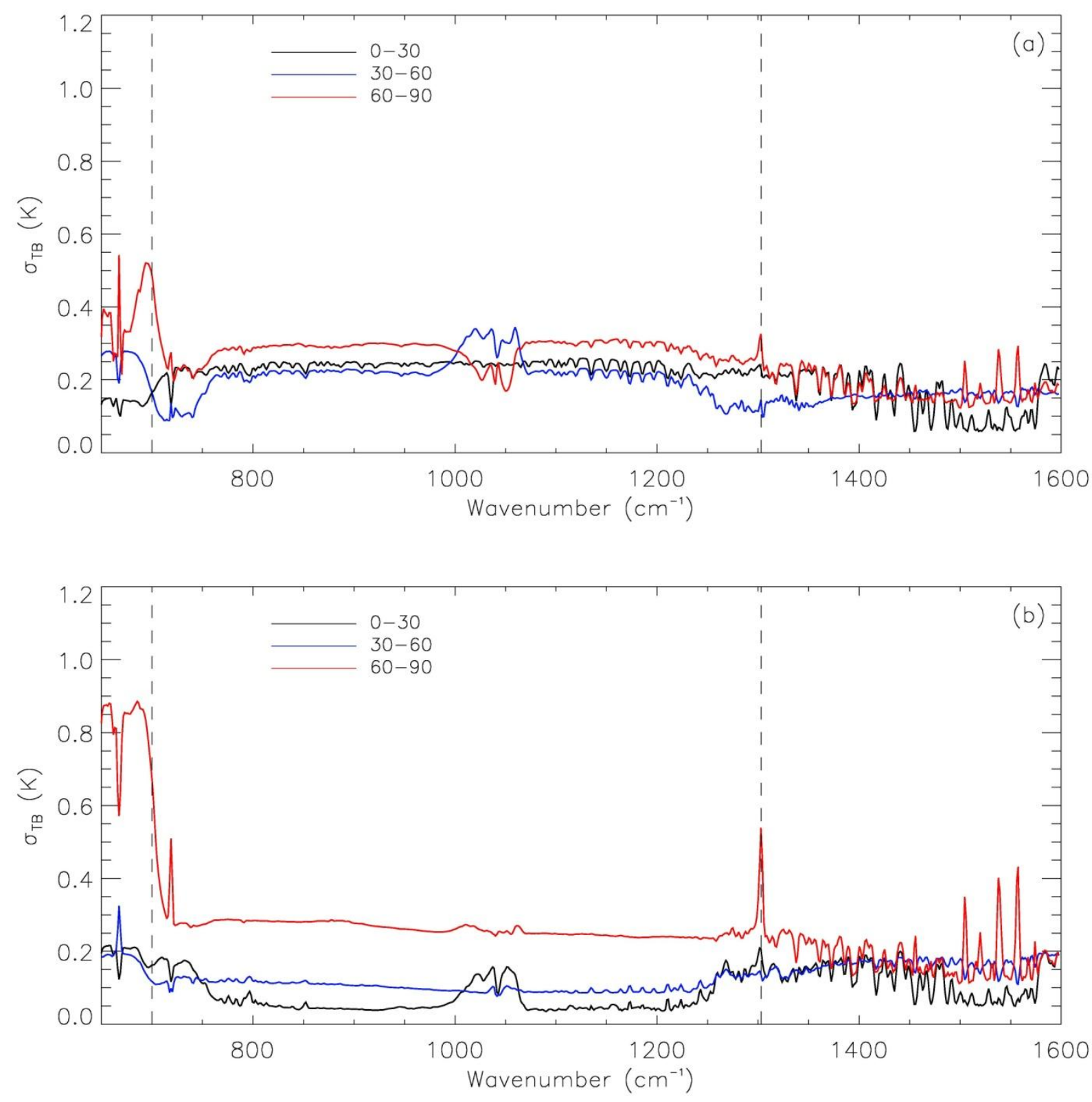

598

599 Figure 3: As Figure 1 for standard deviation in $30^{\circ}$ zonal annual mean spectra

600 

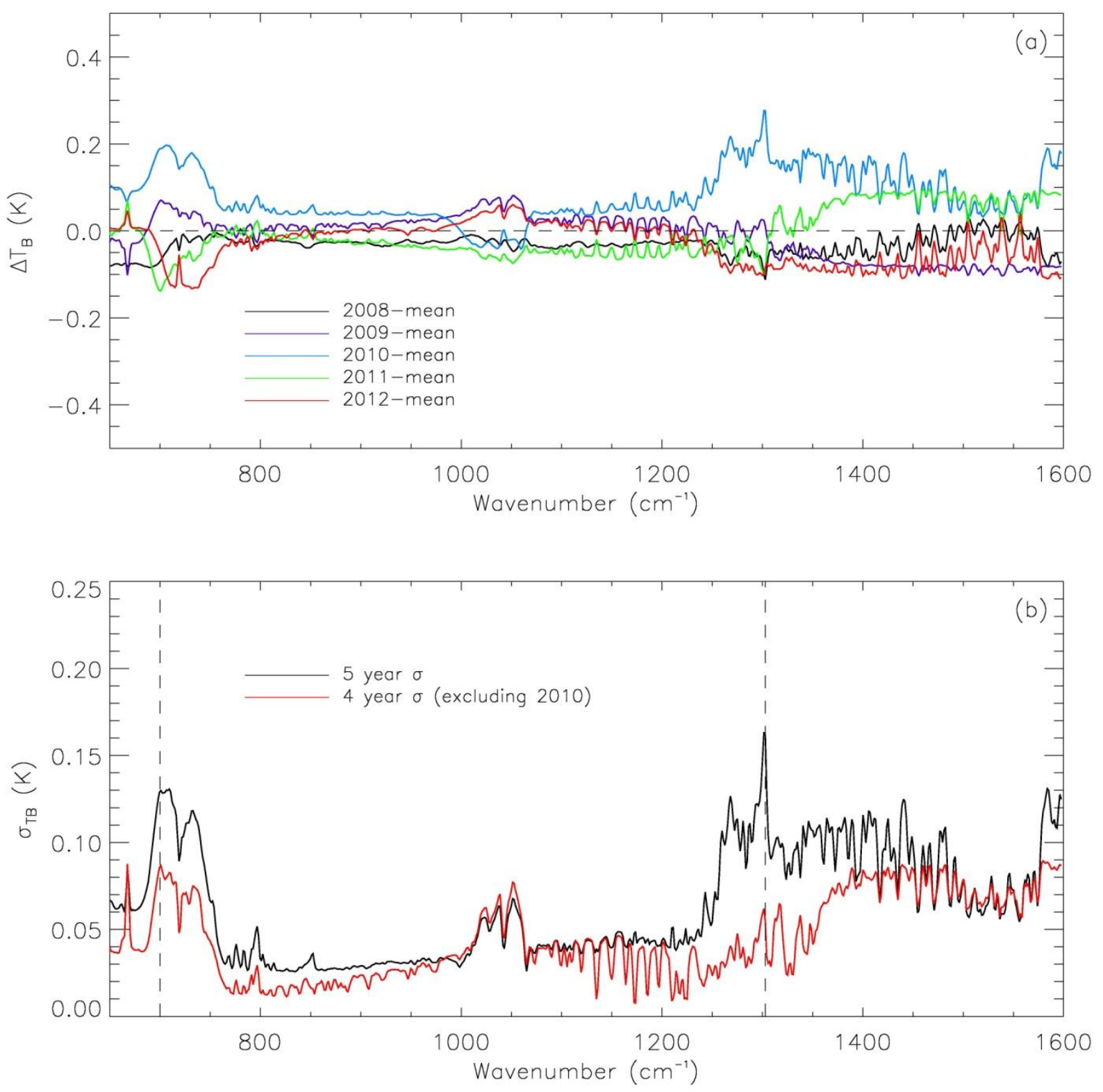

601

602 Figure 4: (a) Deviation in annual global mean brightness temperature from the five year average

603 global annual mean spectrum shown in Fig 1; (b) Standard deviation in global annual mean IRR

604 brightness temperatures for all 5 years (black) and excluding 2010 (red). Dashed vertical lines as 605 in Figures 1 to 3

606 

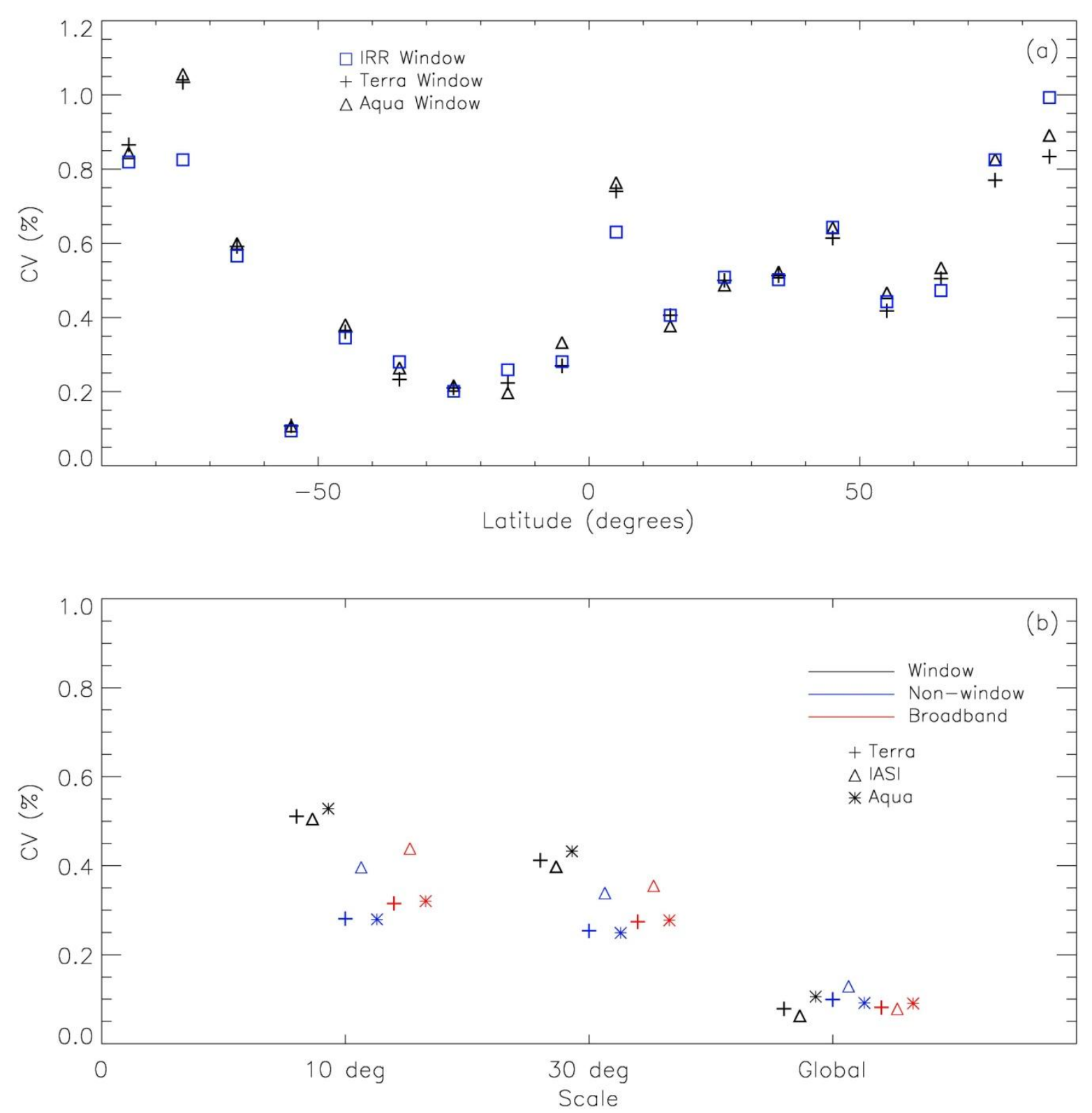

607

608 Figure 5: (a) Coefficient of Variation (CV) in $10^{\circ}$ annual zonal mean window fluxes and 609 radiances measured by CERES and IASI respectively as a function of latitude. (b) Dependence 610 of $\mathrm{CV}$ on spatial scale for different spectral regions and instruments 

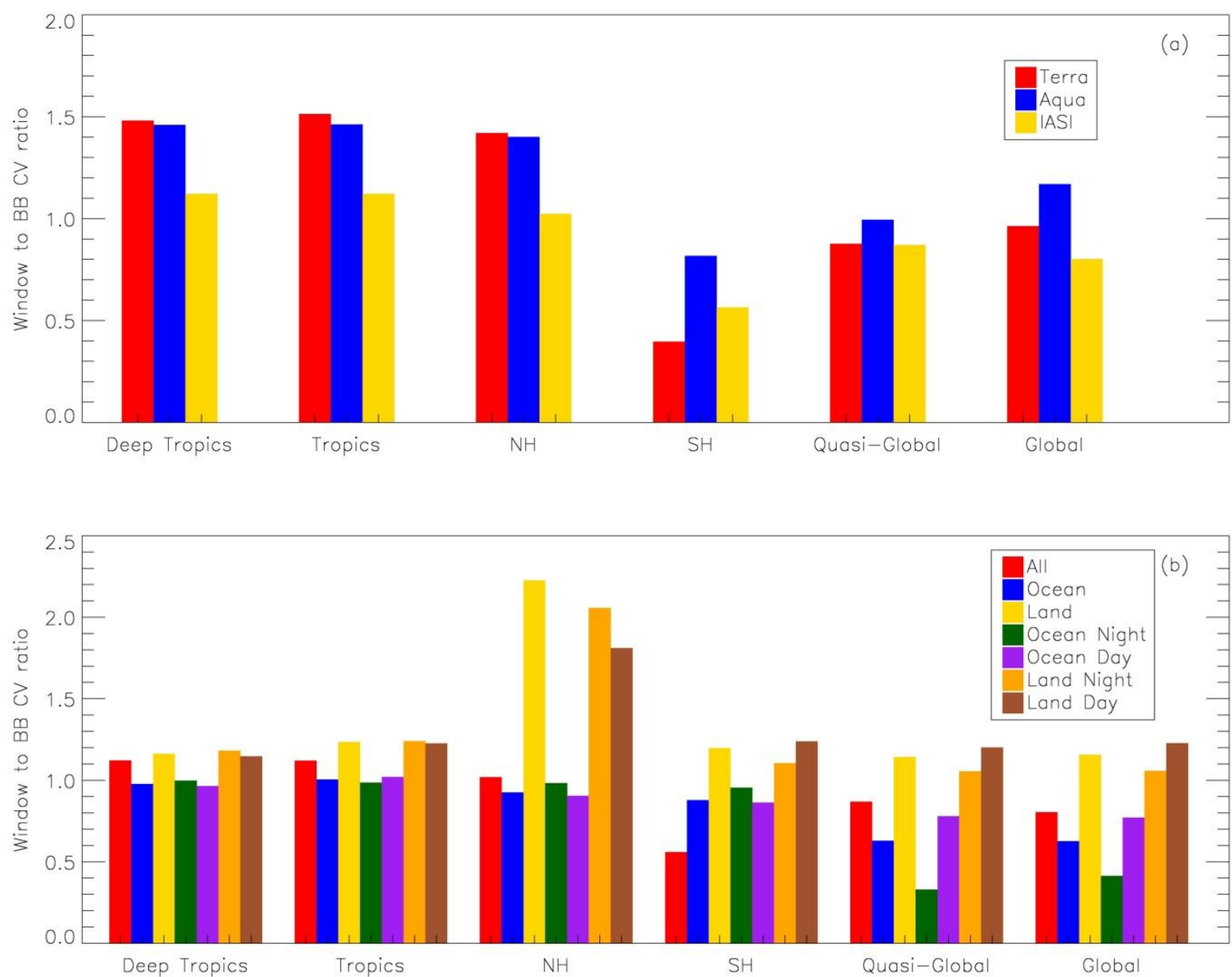

613 Figure 6: Ratio of window to broadband CV for selected regions for (a) Terra, Aqua and IASI 614 (IRR spectra); (b) IASI as a function of sampling characteristics. (Deep-Tropics: $10^{\circ} \mathrm{S}-10^{\circ} \mathrm{N}$; 615 Tropics: $30^{\circ} \mathrm{S}-30^{\circ} \mathrm{N}$; NH: 0-90 $\mathrm{N}$; SH 0-90 ${ }^{\circ} \mathrm{S}$; Quasi Global: $60^{\circ} \mathrm{S}-60^{\circ} \mathrm{N}$ ) 